Revue

de l'histoire des religions
Revue de l'histoire des religions

$4 \mid 2015$

Figures de Noé de Gilgamesh au Coran

\title{
Noé et le Déluge dans la aggada rabbinique
}

Noah and the Flood in Rabbinical Aggada

Jules Danan

\section{OpenEdition}

Journals

Édition électronique

URL : http://journals.openedition.org/rhr/8470

DOI : 10.4000/rhr.8470

ISSN : 2105-2573

Éditeur

Armand Colin

Édition imprimée

Date de publication : 1 décembre 2015

Pagination : 567-584

ISBN : 978-2-200-93012-7

ISSN : 0035-1423

Référence électronique

Jules Danan, "Noé et le Déluge dans la aggada rabbinique », Revue de l'histoire des religions [En ligne], 4 | 2015, mis en ligne le 01 décembre 2018, consulté le 03 mai 2019. URL : http://

journals.openedition.org/rhr/8470 ; DOI : 10.4000/rhr.8470 


\section{Noé et le Déluge dans la aggada rabbinique}

Cet article se propose de voir comment les rabbins appréhendent le personnage de Noé et la "génération du Déluge». Ils s'interrogent à propos du premier sur les raisons qui ont amené Dieu à le sauver et à propos de la seconde sur ce qui l'a conduite à pécher, sur les fautes qu'elle a commises et sur le choix de Dieu de la châtier par le déluge. Nous verrons enfin comment ils envisagent la destinée humaine après le Déluge. Ce faisant, nous tenterons d'expliquer comment les rabbins interprètent - parfois de manière inattendue - les textes bibliques.

\section{Noah and the Flood in Rabbinical Aggada}

The purpose of this article is to examine the rabbinical interpretation of the figure of Noah as well as "the generation of the Flood". The Sages try to understand the reasons leading God to save Noah, but they are interested as well in the causes leading to this generation's sins and, hence, in God' decision to punish it through the Flood. An additional point discussed in this paper is the rabbinical approach toward human destiny after the Flood. The analysis of these texts demonstrates the unexpected manner in which Biblical passages are sometimes interpreted. 
La aggada est ce qui, dans la littérature rabinique, ne concerne pas la halakha, la loi. Elle fait suite aux textes non législatifs de la Bible : récits du Pentateuque, textes prophétiques, historiques, sapientiaux, etc. Elle « complète » le texte dont elle comble les « lacunes », exprime les «non-dits », elle amplifie le texte biblique. Certes, la aggada transmet des récits autres que ceux de la Bible, mais aussi les réflexions des rabbins sur l'éthique, le sens de l'histoire, le sens des lois, le destin de l'homme, etc., en s'efforçant de toujours s'appuyer sur la Bible.

Nous allons essayer de voir dans cet article comment les rabbins appréhendent le personnage de Noé et la « génération du Déluge ». Ils s'interrogent à propos du premier sur les raisons qui ont amené Dieu à le sauver et à propos de la seconde sur ce qui l'a conduite à pécher, sur les fautes qu'elle a commises et sur le choix de Dieu de la châtier par le déluge. Nous verrons enfin comment ils envisagent la destinée humaine après le Déluge.

Les textes que nous allons présenter ici ne constituent pas un ensemble homogène. Ils sont de différentes époques, proviennent de divers recueils, connaissent des variantes, parfois se complètent ou semblent se répondre à travers le temps. Nous avons cependant, pour la clarté du propos, pris le parti d'opérer un classement thématique et de montrer comment les rabbins interprètent - parfois de manière inattendue - les textes bibliques.

\section{Le PERSONNAGe de NoÉ}

\section{Un jugement positif}

La Genèse et la aggada à sa suite portent un jugement positif sur Noé. Il est dit en Gn 6, 8-9 : « Mais Noé trouva grâce aux yeux de l'Éternel. [...] Noé était un homme juste, intègre en sa génération; il marchait avec Dieu » et en Gn 7, 1: «[...] car c'est toi que j'ai reconnu juste parmi cette génération. » Selon Rabbi El'azar ben 'Azaria ( ${ }^{\mathrm{er}}-\mathrm{II}^{\mathrm{e}} \mathrm{s}$.), la différence entre Gn 6,9 (« un homme juste, intègre ») et 7,1 (uniquement «juste ») s'explique par le fait qu'on ne dit jamais tout le bien qu'on pense d'une personne en sa présence, mais hors de sa présence ${ }^{1}$.

1. Genèse Rabba (GnR) 32, 3 (p. 290-291). Genèse Rabba est un recueil d'interprétations (midrash) sur la Genèse compilé au v siècle. Sauf indication 
La perfection morale de Noé va de pair avec sa perfection physique. Le Midrash Tanhuma ${ }^{2}$ affirme en effet que Noé est né circoncis en s'appuyant sur le terme tamim (« intègre », mais aussi " parfait », « sans défaut ») employé en Gn 6, 9 et qui apparaît aussi en Gn 17, 1 dans le contexte du commandement de la circoncision donné à Abraham³ .

L'affirmation de la Genèse « Noé était un homme juste, intègre en sa génération » est amplifiée par les rabbins qui ajoutent en substance : «a fortiori dans d'autres générations », comme celle de Moïse. En d'autres termes, si Noé a pu rester juste dans une génération aussi perverse, a fortiori l'aurait-il été dans une génération vertueuse $e^{4}$.

L'opposition entre Noé et ses contemporains est illustrée par plusieurs textes dont nous citerons deux exemples. Les rabbins sont d'avis que les deux premiers versets du psaume I font référence à Noé. «Heureux l'homme » renvoie en effet à Noé qui «était un homme juste». "Qui ne suit pas les conseils des méchants, qui ne se tient pas dans la voie des pécheurs et ne prend point place dans la société des railleurs », il s'agit là des générations d'Énosh', du Déluge et de la tour de Babel. «Mais qui trouve son plaisir

contraire, nous citerons l'édition critique de J. Theodor et Ch. Albeck, Jérusalem, Shalem Books, 1996. Voir aussi l'édition Romm, Vilna (nombreuses réimpressions) et la traduction française de Bernard Maruani et Albert Cohen-Arazi, Lagrasse, Verdier (coll. « Les Dix Paroles »), 1987.

2. Midrash Tanhuma (Tan) Bereshit 11 et Noah 5. Le Midrash Tanhuma est un recueil d'interprétations sur le Pentateuque du VII ${ }^{\text {e }}$ siècle environ. Salomon Buber a, à partir d'un manuscrit d'Oxford, publié à Vilna en 1885 une édition qui présente des différences avec le Tanhuma « courant»; nous la désignerons par TanB.

3. Pour les rabbins, en effet, la circoncision permet de parfaire le corps de l'homme.

4. Il s'agit là de l'enseignement de Rabbi Nehemia (milieu du II ${ }^{\mathrm{e}} \mathrm{s}$.) dans GnR 30, 9 (p. 276) et Tan Noaḥ 5 et repris par Resh Laqish (III ${ }^{\mathrm{e}}$ s.) dans Sanhedrin 108a.

5. La génération d'Énosh est généralement considérée par les rabbins comme idolâtre. L'expression de Gn 4, 26 «On commença alors à invoquer le nom de l'Éternel » est interprétée « On commença alors à donner le nom de l'Éternel [aux hommes et aux idoles] », le verbe hébraïque utilisé liqro ayant ces deux sens. Pour les châtier, Dieu éleva le niveau de l'océan pour les noyer. Ce châtiment qui présente une certaine analogie avec le déluge est justifié par Am 5, 8 «Il appelle les eaux de la mer et les répand à la surface de la terre, Éternel est son nom. » D'autre part, on trouve le même verbe à propos de la génération d'Énosh (huhal en $\mathrm{Gn} 4,26$ ) et de celle du déluge (hehel en Gn 6,1) et qui signifie à la fois «commencer» et "profaner». Voir en autres le targum attribué à Yonatan ben 'Uzziel sur Gn 4, 26; Mekhilta de-Rabbi Yishma'el (midrash sur l'Exode rapportant les enseignements des tanna'im, rabbins des $\mathrm{I}^{\mathrm{er}}$ et II ${ }^{\mathrm{e}}$ siècles), massekhta 
dans la Torah de l'Éternel », ce sont les sept commandements qu'il a reçus ${ }^{6}$. «Et la médite jour et nuit », ce qui lui a permis de comprendre que si Dieu lui avait ordonné de prendre dans l'arche plus d'animaux purs qu'impurs, c'était pour qu'il puisse offrir un sacrifice après le Déluge ${ }^{7}$.

Job 24, 18 est également mis en rapport avec la génération du Déluge. Genèse Rabba ${ }^{8}$ interprète ainsi ce verset : "Ils sont une épave légère flottant sur l'eau », ils ont été condamnés à périr par l'eau'. «Leur sort est maudit sur terre», [on dira :] «que celui qui a châtié la génération du Déluge [châtie celui qui ne tient pas parole] $»^{10}$. «Ils cessent de prendre le chemin des vignes », leur intention n'était pas de planter des vignes, ce qui veut dire selon les commentateurs, qui se réfèrent au Talmud de Jérusalem ${ }^{11}$, qu'ils ne voulaient que jouir des plaisirs sexuels sans avoir d'enfants. À l'inverse, Noé, lui, voulait crôtre et multiplier puisqu'il est dit : «Voici la descendance (toledot) de Noé » (Gn 6,9). Le Talmud de Babylone ${ }^{12}$ donne une interprétation légèrement différente de ce verset : Noé mettait en garde ses contemporains et les avertissait que Dieu allait envoyer le déluge et que leurs cadavres allaient alors flotter comme des outres gonflées d'air ; la réponse était : « Nous ne quitterons pas le chemin des vignes », ce qui, selon Rashi, signifie : « Nous ne prendrons pas le droit chemin ».

Noé est donc présenté comme un annonciateur, un héraut ${ }^{13}$, dont on rejette les avertissements et les remontrances. Or, rien dans le

$\overline{\text { de-ba-hodesh }}$ 6, éd. H. S. Horovitz et I. A. Rabin, Jérusalem, Shalem Books, 1997 (p. 223) ; GnR 23, 6-7 (p. 227-229).

6. Ce sont l'obligation d'établir des tribunaux, l'interdiction de l'idolâtrie, du blasphème, de l'inceste et de l'adultère, du meurtre, du vol, de la consommation d'un membre arraché à un animal vivant. On les appelle aussi les sept commandements des Fils de Noé auxquels, selon les rabbins, sont astreints tous les êtres humains, les «Fils de Noé ». Voir Tosefta 'Avoda zara 8, 4 (éd. Zuckermandel, Jérusalem, Wahrmann Books, 1970, p. 473) et Sanhedrin 56a.

7. GnR 26, 1 (p. 243-244); Midrash Tehillim sur Ps 1 (éd. Salomon Buber, Jérusalem, 1977, p. 11). Pour le sacrifice de Noé, voir Gn 8, 20.

8. GnR 30, 2 (p. 270-271).

9. Un commentaire de GnR attribué à Rashi (voir l'édition Romm) justifie cette interprétation en rapprochant qal, « léger» en hébreu, de $\mathrm{Jb} 24,18$ de son homonyme araméen présent dans Dn 4, 28 où il a le sens de voix céleste.

10. Cf. Bava mets' ia 4, 2.

11. TJ Yevamot 6, 5 (7c).

12. Sanhedrin 108a-b.

13. GnR 30, 7 (p. 273). 
récit de la Genèse ne permet d'attribuer un tel rôle à Noé. D'autres textes bibliques sont invoqués pour le justifier dont Am 5, 10 : « Ils haïssent quiconque réprimande à la Porte, ils abhorrent celui qui parle avec intégrité (dover tamim) ». Cet homme est bien sûr Noé que la Bible qualifie de tamim $(\mathrm{Gn} 6,9)^{14}$.

Noé a mis les 120 ans mentionnés en Gn 6, 3 - et que les rabbins considèrent comme le délai que Dieu a accordé aux hommes avant le Déluge - à planter des cèdres, les arroser, les couper, les scier pour construire l'arche et à annoncer le Déluge. Mais ses contemporains se moquaient de lui, le traitaient de vieillard méprisable, prétendaient que le déluge s'abattrait sur sa famille uniquement, affirmaient qu'ils avaient les moyens de se protéger de ce déluge quel qu'il fût : feu, eau du ciel, eau souterraine ${ }^{15}$.

Un autre point est absent de la Genèse mais que les rabbins mentionnent : les bienfaits que Noé aurait apportés aux hommes. Il est dit en Gn 5, 28-29 : "Lemekh vécut 182 ans et engendra un fils. Il l'appela Noé, en disant : 'celui-ci nous consolera de notre tâche et du labeur de nos mains, causé par la terre que l'Éternel a maudite'. » Comment, se demande le Midrash Tanhuma ${ }^{16}$, Lemekh pouvait-il savoir que l'enfant qui lui était né allait les consoler? C'est que Noé est né circoncis et c'était là le signe que Dieu avait donné à Adam pour la fin de la malédiction de la terre ( $\mathrm{Gn}$ $3,17)$. Avant la naissance de Noé, on ne récoltait pas ce qu'on plantait. On plantait du blé et on moissonnait des ronces $(\mathrm{Gn} \mathrm{3,18).}$ Après la naissance de Noé, le monde a recommencé à fonctionner normalement : on moissonnait ce qu'on semait. De plus, jusque-là, les hommes travaillaient la terre de leurs mains, au sens propre du terme, Noé leur a apporté la charrue, la faucille, la pioche et tous les autres outils.

Dans Genèse Rabba ${ }^{17}$, on se pose la question de l'adéquation du nom de Noé - Noah, d'une racine signifiant repos - à l'étymologie qu'en donne la Genèse «celui-ci nous consolera ». Pour Rabbi Yoḥanan (c. 180-279), lorsque Dieu a créé Adam, il l'a fait

14. GnR 31, 3 (p. 278-279).

15. GnR 30, 7 (p. 272-273) ; Sanhedrin 108a-b ; Tan Noah 5 ; TanB Bereshit 37 (p. 25).

16. Tan Bereshit 11.

17. GnR 25, 2 (p. 239-240). 
maître du monde : la vache obéissait au laboureur et la porte au charpentier ${ }^{18}$. Après la faute, ces éléments se sont révoltés contre l'homme et ont cessé de lui obéir. Avec Noé, ils se sont « reposés » de leur révolte et les hommes ont été consolés de leurs peines. Rabbi Yohanan justifie ce repos par analogie avec Ex 23, 12 : «afin que ton bœuf et ton âne se reposent ». Pour son contemporain Resh Laqish, avant Noé, l'eau remontait et inondait les sépultures matin et soir. Resh Laqish s'appuie sur une expression de Ps 88, 6 qu'il faut lire, selon son interprétation, «ceux qui sont couchés dans la tombe sont comme des morts [par noyade] » et sur les deux occurrences chez Amos (5, 8 et 9,6) de la phrase «Il appelle les eaux de la mer ». Avec Noé, les morts ont retrouvé le repos ainsi que le dit Isaïe $(57,2)$ : « Ils entrent dans la paix, reposent sur leurs couches. »

\section{Un jugement relativisé}

Cependant, le jugement porté sur Noé n'est pas entièrement favorable. Il est intéressant de constater que les textes qui donnent une image positive de Noé peuvent être également interprétés de manière négative. Ainsi peut-on lire dans Genèse Rabba à propos de Noé « homme juste, intègre en sa génération » $(\mathrm{Gn} 6,9)$ : «Dans sa génération, c'était un juste, mais il ne l'aurait pas été s'il avait appartenu à la génération de Moïse ou de Samuel. Sur la place des aveugles, on appelle le borgne 'bien voyant' et le petit, 'Maître' ${ }^{19}$.

Noé est considéré comme moins «solide » qu'Abraham. Noé «marchait avec Dieu» $(\mathrm{Gn} 6,9)$, tandis que Dieu dit à Abraham : «marche devant moi » $(\mathrm{Gn} 17,1)$, fait remarquer Rabbi Yehuda ${ }^{20}$. En quelque sorte, Dieu soutenait Noé pour qu'il ne s'enfonce pas dans la perversion de la génération du Déluge, comme un père soutient son fils pour qu'il ne s'enfonce pas dans la boue. Abraham

18. «Le sillon au laboureur » selon l'édition Romm. La version rapportée par le Yalqut Shim 'oni (anthologie de textes rabbiniques sur la Bible, XIII' ${ }^{\text {e s.) }} 42$ est : «le sillon se laissait creuser».

19. GnR 30, 9 (p. 275) et Tan Noaḥ 5 citent Rabbi Yehuda (milieu du II ${ }^{\mathrm{e}}$ s.) auquel s'oppose, nous l'avons vu plus haut, son contemporain Rabbi Nehemia. La même opposition se retrouve dans Sanhedrin 108a entre Rabbi Yohanan qui reprend les propos de Rabbi Yehuda et son disciple, puis collègue, Resh Laqish. Voir ci-dessus note 4.

20. GnR 30, 10 (p. 276). 
et les patriarches ouvraient la voie à Dieu, allaient devant lui pour accomplir sa volontée ${ }^{1}$.

Plus grave encore, Noé n'aurait pas mérité d'être sauvé, mais il « a trouvé grâce aux yeux de l'Éternel » $(\mathrm{Gn} 6,8)$. Cet enseignement de l'école de Rabbi Yishma'el ( $1^{\text {re }}$ moitié du II ${ }^{\mathrm{e}} \mathrm{s}$.), repris par Rabbi Abba bar Kahana (fin du III ${ }^{\mathrm{e}} \mathrm{s}$.) se fonde sur le waw hébraïque, « et » ou «mais », qui relie les versets 7 et 8 du chapitre VI. Une double lecture est alors possible : «[...] car je regrette de les avoir faits et Noé [aussi] » et «[...] car je regrette de les avoir faits. Mais Noé a trouvé grâce aux yeux de l'Éternel $»^{22}$. Et s'il a bénéficié de la miséricorde divine, c'est par le mérite de ses descendants. En effet, «Mais Noé a trouvé grâce aux yeux de l'Éternel » $(\mathrm{Gn} 6,8)$ est immédiatement suivi de «Voici la descendance de Noé $»^{23}$. Et c'est parce que Moïse devait descendre de Noé que celui-ci a été épargné ${ }^{24}$. Selon les rabbins en effet, le monde n'a été créé que pour que la Torah soit donnée aux hommes, il ne subsiste que par l'étude de celle-ci ${ }^{25}$. Moïse est d'ailleurs préféré à Noé puisqu'il est d'abord désigné comme « un homme égyptien» (Ex 2,19) avant d'être nommé «l'homme de Dieu » (Dt 33,1) alors que Noé n'est plus que « l'homme de la terre » $(\mathrm{Gn} 9,20)$ après avoir été « un homme juste » $(\mathrm{Gn} 6,9)^{26}$.

Enfin, le Noé qui dans la Genèse obéit scrupuleusement aux ordres de Dieu est qualifié d' « incroyant » par Rabbi Yoḥanan qui affirme que Noé n'est entré dans l'arche que lorsqu'il a eu de l'eau aux chevilles. En effet, « Noé entra [...] dans l'arche en raison des eaux du déluge » $(\mathrm{Gn} 7,7)^{27}$.

L'image de Noé que reflète la aggada est donc ambivalente. Homme juste, c'est par lui que le monde a été [re]créé ${ }^{28}$. Il a

21. Tan Noah 5.

22. Sanhedrin 108a; GnR 28, 9 (p. 267).

23. GnR 29, 5 (p. 270); Tan Noah 2.

24. GnR 26, 6 (p. 253). Selon ce texte, les cent vingt ans mentionnés en Gn 6,3 sont une allusion à Moïse dont c'était les années de vie (cf. Dt 31,2).

25. Voir par exemple GnR 1, 4 (p. 7); Shabbat 48a ; Pesahim 68b; 'Avoda zara $3 \mathrm{a}$.

26. GnR 36, 3 (p. 337). Il faut cependant noter que l'expression « homme de la terre » n'est pas uniquement considérée comme négative. Voir aussi TanB Noah 20 (p. 46).

27. GnR 32, 6 (p. 293). Le mot mippene, «à cause de, en raison de» peut également se traduire « devant».

28. Tan Bereshit 11. Une autre version, rapportée par Rashi dans son commentaire sur Gn 5, 28, serait «le monde a été [re]construit par lui ». Cette formulation s'appuie sur le fait que dans la généalogie de Gn 5, le terme ben 
façonné la terre par ses cultures et l'a repeuplée ${ }^{29}$. Mais d'autres considèrent que ce n'est pas un juste parfait, que sa foi n'est pas totale. Il s'est «profané ${ }^{30}$ en plantant une vigne et s'est avili en s'enivrant ${ }^{31}$. Certes, la Genèse se montre déjà critique envers Noé lorsqu'elle rapporte l'épisode de la vigne, mais l'image négative de Noé permet aussi de mettre en valeur les patriarches d'Israël et Moïse qui, lui aussi, a survécu grâce à une arche ${ }^{32}$. Cependant, même si Noé n'est pas considéré comme un juste parfait, on ne lui reproche en rien les fautes commises par ses contemporains.

\section{LE DÉLUGE : FAUTES ET CHÂTIMENT}

\section{Les fautes}

La Genèse invoque deux raisons à la décision de Dieu de détruire le monde : la dépravation et la violence. Le texte biblique est cependant concis et la aggada va l'amplifier et donner force détails sur les fautes commises par les hommes et qui ont entraîné leur destruction par le déluge.

La perversion sexuelle de la génération du Déluge est justifiée dans le Talmud ${ }^{33}$ par Gn 6,12: «Dieu vit que la terre était corrompue, car toute chair avait corrompu sa voie sur la terre. » Les rabbins relient à ce verset le récit des bne ha-Elohim «les fils de Dieu » et des «filles de l'homme» (Gn. 6, 1-4). Une littérature nombreuse considère qu'il s'agit là d'anges déchus ${ }^{34}$ et l'on trouve également cette conception dans des écrits rabbiniques ${ }^{35}$. Cependant, les

«fils»- proche de la racine bnh « construire »- n'apparaît qu'à propos de la naissance de Noé.

29. GnR 36, 3 (p. 337).

30. Il y a là un jeu de mots entre wa-yahel « commencer » $(\mathrm{Gn} 9,20)$ et nithallel « devenir profane ». Voir également ci-dessus note 5.

31. GnR 36, 3 et 4 (p. 337 et 338).

32. Dans le texte hébraïque, le même mot - teva «caisse, boîte »- est utilisé à propos de Noé $(\mathrm{Gn} 6,14)$ et de Moïse $(\operatorname{Ex} 2,3)$. Voir GnR 31, 9 (p. 282) où est expliquée la différence entre les produits utilisés pour imperméabiliser les deux arches.

33. Sanhedrin $57 \mathrm{a}$.

34. Voir à ce sujet Louis Ginzberg, Les Légendes des Juifs, trad. Gabrielle SedRajna, Paris, Cerf, 1997, t.1 p. 93 et 268 n. 57.

35. Voir par exemple Yalqut Shim'oni 44 ; Pirqe de-Rabbi Eli'ezer (ouvrage du VIII ${ }^{\mathrm{e}}$ s.), chap. 22 (Chapitres de Rabbi Eliezer, trad. Marc-Alain Ouaknin et Eric Smilévitch, Lagrasse, Verdier, 1983). 
textes rabbiniques anciens préfèrent voir en ces «fils d'Élohim» des hommes et non des êtres surnaturels. Pour Rabbi Shim'on ben Yohaï (milieu du $\mathrm{II}^{\mathrm{e}} \mathrm{s}$.), ce sont les « fils des juges $»^{36}$. On retrouve cette idée dans le targum araméen qui traduit «les grands » ou plus tard chez Rashi qui parle des « fils des princes et des juges ». Le même Rabbi Shim'on poursuit : "Toute brèche qui n'est pas [ouverte] par les grands n'en est pas une. » Ce sont les «grands » qui les premiers ont péché, suivis en cela par les autres hommes.

Selon un passage de Genèse Rabba, les manifestations de cette perversion sont précisées dans Gn 6, 2 interprété ainsi : "'Les fils de Dieu virent que les filles de l'homme étaient belles', il s'agit des vierges ; lorsqu'on parait [une jeune fille] pour son époux, un grand entrait et la possédait en premier. 'Ils prirent des femmes', ce sont les femmes mariées. 'Parmi tout ce qu'ils choisirent', ce sont les hommes et les animaux; la génération du Déluge n'a été effacée de l'univers qu'après qu'on eut établi des contrats de mariage entre hommes et avec des animaux $»^{37}$.

Les femmes aussi ont péché. En effet, « lorsqu'une femme sortait et voyait un jeune homme qu'elle désirait, elle avait ensuite un rapport [avec son époux] et donnait naissance à un jeune homme semblable $»^{38}$.

Les animaux ont également contribué à la faute ainsi que le précise le texte biblique : "car toute chair avait corrompu sa voie sur la terre » $(\mathrm{Gn} 6,12)^{39}$. Ils ont enfreint la volonté de Dieu qui les a créés « selon leurs espèces » $(\mathrm{Gn} 1,25)^{40}$ et se sont accouplés hors de leur espèce : le chien avec le loup, le coq avec le paon ${ }^{41}$,

36. GnR 26, 5 (p. 247). Pour les rabbins, Elohim «Dieu, les dieux » désigne aussi les juges comme dans Ex 22, 8. Lorsqu'il s'agit de Dieu, le nom Elohim renvoie à l'attribut de justice. L'expression « fils de Dieu » est justifiée dans ce même passage de Genèse Rabba par le fait que ces hommes ont vécu longtemps sans connaître de souffrance. Ils ont bénéficié de cette longévité pour qu'ils puissent observer les astres et transmettre leur savoir. Selon le texte publié par Salomon Buber dans l'introduction (p. XXXVIII) à son édition de Aggadat Bereshit (Krakau, 1902), il s'agit des descendants de Caïn dont il est dit : «J'ai créé un homme avec l'Éternel » $(\mathrm{Gn} 4,1)$.

37. GnR 26, 5 (p. 248) ; la traduction de Gn 6, 12 tient compte de l'interprétation du verset dans ce passage. Voir aussi TanB Bereshit 33 (p. 23-24), Sanhedrin 108a.

38. GnR 26, 7 (p. 254). Voir aussi TanB Bereshit 40 (p. 26).

39. GnR 28, 8 (p. 266); Sanhedrin 108a ; Tan Noah 12.

40. Tan Noah 5, TanB Noah 5 (p. 31).

41. GnR 28, 8 (p. 266). 
le cheval avec l'âne, le serpent avec l'oiseau ${ }^{42}$. Selon le Talmud de Babylone ${ }^{43}$, la responsabilité de cet état de fait incombe aux hommes qui ont opéré des croisements entre les espèces animales. Ce texte laisse supposer que les animaux se sont ensuite accouplés d'eux-mêmes hors de leur espèce puisqu'il précise que les animaux, à une exception près ${ }^{44}$, ont recommencé après le déluge à s'accoupler à l'intérieur de leurs espèces. Les animaux sauvés du déluge n'étaient pas pervertis. Cette affirmation s'appuie sur Gn $7,2:$ : $[\ldots]$ tu prendras $[\ldots]$ un mâle et sa femelle $[\ldots] »^{45}$, ou encore sur Gn 8,19: « Tous les animaux [...], selon leurs familles, sont sortis de l'arche $»^{46}$.

La Genèse $(6,11$ et 13$)$ reproche également à la génération du Déluge de se livrer au hamas, terme généralement traduit par «violence » ou par «iniquité ». Cependant, les rabbins y voient plutôt le vol, gazel, dont la gravité est soulignée par Rabbi Yoḥanan qui affirme : «La génération du Déluge a commis toutes les fautes, mais son jugement n'a été scellé que lorsqu'elle s'est livrée au vol (gazel) ainsi qu'il est dit : 'car la terre est remplie de violence (hamas) à cause de leurs actes et je vais les détruire avec la terre' $(\mathrm{Gn} 6,13) »^{47}$. Genèse Rabba parle de vol par effraction ${ }^{48}$, mais donne surtout l'exemple suivant :

Les hommes de la génération du Déluge agissaient ainsi : l'un d'entre eux présentait [sur le marché] son panier de lupins. Un homme venait et soustrayait [une quantité] inférieure à la valeur d'une pruța ${ }^{49}$. Chacun faisait de même, de sorte que [le marchand, ayant perdu toute sa marchandise] ne pouvait demander réparation ${ }^{50}$. Le Saint béni soit-il dit : « Vous avez agi en dehors de la norme, j' agirai aussi contre vous en dehors de la norme $»^{51}$.

Le hamas est également identifié à l'effusion de sang par analogie avec Jl 4, 19: «L’Égypte sera une solitude, Édom un désert délaissé,

42. Tan Noah 12.

43. Sanhedrin 108a.

44. Le tushalmi, considéré comme une sorte d'oiseau.

45. Littéralement « un homme et sa femme ». Sanhedrin $108 \mathrm{~b}$.

46. Tan Noah 12 ; TanB Noah̆ 5 (p. 31).

47. Sanhedrin 108a. Voir aussi GnR 31, 1-4 (p. 277-279) et Tan Noah 4.

48. GnR 27, 3 (p. 257).

49. Monnaie de peu de valeur.

50. La valeur du larcin étant minime, aucun n'est justiciable.

51. GnR 31, 5 (p. 279). 
en raison de la violence (hamas) exercée sur les fils de Juda dont ils ont versé le sang innocent dans leur pays $»^{52}$.

En plus de la perversion sexuelle et de la violence dont parle le Pentateuque, les textes rabbiniques imputent à la génération du Déluge des fautes contre Dieu. C'est ainsi que le Talmud de Babylone $^{53}$ considère que les hommes se livraient à l'idolâtrie et le justifie en rapprochant Gn 6, 11 : «La terre se pervertit devant Dieu » de Dt 4, 16 : «De crainte que vous vous pervertissiez en fabriquant des idoles $»^{54}$.

À l'idolâtrie s'ajoutent l'orgueil et le blasphème. Les bienfaits décrits dans $\mathrm{Jb} 21,8-13$ ont été accordés, selon les rabbins, à la génération du Déluge qui, pourtant, a dit (versets 14-15) « à Dieu : 'Éloigne-toi de nous, nous ne voulons pas connaître tes voies. Qu'est-ce que le Tout-Puissant pour que nous le servions? Quel profit aurions-nous à lui adresser nos prières ?» Ce qui signifie : « Nous n'aurions besoin de lui que pour la pluie [qu'il prodigue], [mais] nous avons des rivières et des sources dont nous nous satisfaisons $»^{55}$.

Pour Rabbi Yehuda ha-nasi ${ }^{56}$, les hommes contestaient à Dieu la capacité de les juger et de les punir. Il adopte pour justifier cette idée une lecture particulière du début de Gn 6,3 . Ce passage est diversement traduit ou expliqué : «L'Éternel dit : 'Mon esprit n'animera plus les hommes' ${ }^{57}$, "Yahvé dit : 'Mon esprit ne demeurera pas dans l'homme' $»^{58}, \ll$ Le Seigneur dit : 'Mon Esprit ne dirigera pas toujours l'homme' ${ }^{59}$, « L'Éternel dit : 'Cette génération perverse ne perdurera pas devant moi' $»^{60}$. Rashi, pour sa part, explique : «Mon esprit ne va pas argumenter et disputer à cause de

52. GnR 31, 6 (p. 280). Voir aussi Bava qamma 119a, Tan Noah 4.

53. Sanhedrin 57a.

54. Dans GnR 31, 6 (p. 280), le hamas est identifié à l'idolâtrie par référence à Gn 6,13, mais différents commentateurs considèrent qu'il faut plutôt se référer à Ez 8,17 où le terme hamas est associé à des pratiques idolâtres.

55. Sanhedrin 108a; Tosefta Sota 3, 6-7 (p. 296); Mekhilta de-Rabbi Yishma'el, massekhta de-shira 2 (p. 121-122).

56. Président (nasi) du Sanhedrin et Patriarche de Judée de 170 à 220. On lui doit la rédaction de la Mishna.

57. Traduction des membres du rabbinat français sous la direction de Zadoc Kahn.

58. La Bible de Jérusalem.

59. Traduction œcuménique de la Bible.

60. Targum d'Onqelos. 
l'homme. Mon esprit débat en moi [pour savoir s'il faut les] détruire ou [les] prendre en pitié, cette querelle dans mon esprit ne durera pas. » Rabbi Yehuda ha-nasi propose donc la lecture suivante : «La génération du Déluge a dit : 'L'Éternel ne me jugera pas' ${ }^{61}$. ${ }^{62}$ Dans le texte hébraïque, le sujet Adonaï est placé après le verbe wa-yomer, ce qui permet à Rabbi Yehuda ha-nasi d'introduire un nouveau sujet (la génération du Déluge) et de considérer la suite du texte comme la parole prononcée par les hommes. Cette interprétation va dans le sens de l'enseignement de Rabbi Akiba (c. 50-135) qui cite Ps 10, 13 : « Pourquoi le méchant outrage-t-il Dieu, dit-il : 'tu ne demandes aucun compte' » et explique que les méchants affirment qu'il n'y a ni justice ni juge, mais il y a bien une justice et un juge ${ }^{63}$.

\section{Le châtiment}

«Rabbi El'azar (III ${ }^{\mathrm{e}} \mathrm{s}$.) dit : 'lorsqu'il n'y a pas justice [ici-bas], il y a justice [là-haut]'. [...] Rabbi Me'ir (II s.) dit : 'ils n'ont pas appliqué la justice ici-bas, ne l'appliquerai-je pas là-haut ?' ${ }^{64}$ Les hommes n'ayant obéi à aucune loi, n'ayant pas fait régner la justice, le châtiment divin s'abat sur eux. Les rabbins s'interrogent sur la décision de Dieu de détruire la terre par le déluge. Selon Rabbi Levi ( $\mathrm{III}^{\mathrm{e}}$ s.) dont l'enseignement est rapporté par Rabbi Abba bar Kahana, le monde revient ainsi à son état antérieur, avant la création. Cette idée est illustrée par la parabole suivante :

Un roi fit construire un palais et y installa des habitants muets. Tous les matins, ceux-ci saluaient le roi en faisant des signes avec les doigts ou [en agitant] leurs écharpes. Le roi dit : «Si ces habitants qui sont muets me saluent tous les matins par signes, qu'en serait-il s'ils étaient doués de parole ! » Il installa dans le palais des hommes doués de parole, mais ceux-ci s'emparèrent du palais et se révoltèrent contre le roi en disant : « Ce palais nous appartient ! » Le roi dit alors : «Que ce palais redevienne ce qu'il était!» Ainsi, au commencement la louange adressée au Saint béni soit-il ne venait que des eaux ainsi qu'il est écrit : « [Plus que les] voix des eaux nombreuses, des vagues impétueuses de la mer» (Ps 93, 4) et que disaient-elles? « Puissant est l'Éternel dans les hauteurs ». Le Saint béni soit-il dit : «Si ces

61. Littéralement «ne jugera pas mon âme». Le mot ruah signifie à la fois « esprit, âme, souffle».

62. GnR 26, 6 (p. 252).

63. Ibid.

64. Ibid. Rabbi Me'ir fait ici parler Dieu. Cela est courant dans les textes rabbiniques, nous en verrons d'autres exemples plus loin. 
[eaux] qui n'ont ni bouche ni faculté de parler [agissent] ainsi, qu'en sera-t-il lorsque je créerai l'homme ! » La génération d'Énosh s'est révoltée [contre Dieu], de même que celle du Déluge et de la tour de Babel. Le Saint béni soit-il dit : «Que disparaissent celles-ci et que reviennent celles-là », ainsi qu'il est écrit : «L'Éternel dit : je vais effacer l'homme $»(\mathrm{Gn} 6,7)^{65}$.

Pour Rabbi Berekhia (IV ${ }^{\mathrm{e}} \mathrm{s}$.), l'eau du déluge a effacé la poussière dont est fait l'homme ${ }^{66}$. Tout a été effacé, y compris la dernière vertèbre, considérée comme indestructible, à partir de laquelle chaque être ressuscitera ${ }^{67}$. De ce fait, la génération du Déluge ne ressuscitera pas à la fin des temps ${ }^{68}$.

D'autres raisons sont invoquées qui correspondent à la vision qu'ont les rabbins de la justice divine et qu'ils expriment par les formules : « on mesure à l'homme avec la même unité de mesure qu'il utilise $»^{69}$, $«$ mesure pour mesure ${ }^{70}$, ou encore $«$ il châtie les nations par là où elles se sont montrées hautaines à son égard ${ }^{71}$. En d'autres termes, rétribution ou châtiment divins sont de la même nature que les actions des hommes. Ainsi, les hommes ont dit qu'ils avaient suffisamment d'eau et n'avaient aucunement besoin de la pluie que Dieu pouvait prodiguer, ils ont donc été punis par le déluge ${ }^{72}$.

Les hommes «se sont enorgueillis par le globe de l'œil qui est semblable à l'eau ainsi qu'il est dit 'Les fils de Dieu virent que les filles de l'homme étaient belles' (Gn 6, 2), le Saint béni soit-il les a châtiés par l'eau ainsi qu'il est dit 'en ce jour, jaillirent toutes les sources du grand abîme' ${ }^{73}$. Selon Rabbi Levi, « ils ont perverti leurs canaux $»^{74}$,

65. GnR 28, 2 (p. 260).

66. Ibid. (p. 261).

67. Ibid. 28,3 (p. 261).

68. Sanhedrin 10, 3 et 108a; GnR 26, 6 (p. 249-250) et 28, 9 (p. 266-267) ; TanB Bereshit 40 (p. 26).

69. Par exemple, Soța 1,7.

70. Par exemple, Shabbat 105b.

71. Par exemple, Mekhilta de-Rabbi Yishma'el, massekhta de-shira 2 (p. 121). Cette sentence s'applique aussi à des individus, tel Absalon qui a été puni par sa chevelure dont il s'enorgueillissait (cf. 2 S 14, 25-26 et 18, 9).

72. Ibid. (p. 122) ; Tosefta Sota 3, 8 (p. 296) ; Sanhedrin 108a.

73. Tosefta Sota 3, 9 (p. 296). Il y a ici un jeu de mots entre ma'yanot, « sources », et 'ayin, « œil » mais aussi « source». Voir aussi Sanhedrin 108a et GnR 32, 7 (p. 294). On peut lire dans la Mekhilta de-Rabbi Yishma'el (p. 122) la variante suivante : "Ils ont porté l'œil d'en haut sur l'œil d'en bas pour assouvir leur désir et le Saint béni soit-il a ouvert contre eux les sources d'en haut et d'en bas. »

74. Référence à la perversion sexuelle. 
aussi Dieu a-t-il modifié l'ordre des choses. Habituellement, la pluie tombe et l'eau des profondeurs remonte. Dans le cas du déluge c'est le contraire qui s'est passé : « les sources du grand abîme jaillirent » d'abord puis « les écluses du ciel s'ouvrirent » $(\mathrm{Gn} 7,11)^{75}$.

Les hommes, selon Rav Hisda ( $\mathrm{III}^{\mathrm{e}}-\mathrm{IV}^{\mathrm{e}} \mathrm{s}$.), « ont péché par [un liquide] bouillant ${ }^{76}$ et ils ont été punis par de l'eau bouillante $\gg^{77}$. Il n'y a rien de tel dans la Genèse, cependant Rav Hisda rapproche Gn 8,1 «les eaux s'apaisèrent » d'Est 7, 10 «la colère du roi s'apaisa ». Le même verbe est utilisé dans les deux cas, mais dans le second, il est associé à hema, colère, terme qui évoque aussi la chaleur. Rabbi Yohanan affirme par ailleurs que chaque goutte d'eau du déluge a été chauffée dans la Géhenne ${ }^{78}$ et cite même trois sources chaudes qui ne se sont pas refermées après le déluge ${ }^{79}$.

Enfin, si le déluge a duré quarante jours et quarante nuits $(\mathrm{Gn} 7,4)$, c'est parce que les hommes ont enfreint la Torah donnée au bout de quarante jours ${ }^{80}$ ou encore parce qu'ils ont altéré la forme [de l'embryon] donnée au bout de quarante jours [de grossesse ${ }^{81}$. R. Yohanan semble bien faire ici référence à l'adultère. Son enseignement peut être éclairé par d'autres textes ${ }^{82}$ qui évoquent une femme déjà enceinte de son mari et qui commet un adultère. En quelque sorte, Dieu change la «forme » de l'enfant qu'il a faite à la ressemblance du mari pour la faire à la ressemblance de l'amant. Pour Rashi (sur Gn 7, 4), les hommes ont contraint le Créateur à donner forme à des enfants adultérins.

\section{Après le déluge}

Malgré le châtiment, le monde d'après le Déluge n'est pas foncièrement meilleur. Job 24, 20 est cité dans Genèse Rabba et

75. GnR 32, 7 (p. 294 et note 2) ainsi que l'édition Romm.

76. Allusion au sperme.

77. Sanhedrin $108 \mathrm{~b}$.

78. GnR 28, 9 (p. 267).

79. Sanhedrin $108 \mathrm{a}$.

80. Cf. Ex 24, $18 ; 31,18 ; 34,27-28$; Dt 9, 9-11.

81. GnR 32, 5 (p. 292). Sur la formation de l'embryon, voir Nidda 3, 7 ; Berakhot 60a.

82. Lévitique Rabba 23, 12 (éd. M. Margulies, New York et Jérusalem, 1993, p. 545 et suiv.) ; Tan Naso 4 ; Nombres Rabba 9, 1. 
appliqué à la génération du Déluge : « La matrice [qui les a portés] les a oubliés, la vermine se délecte d'eux, nul ne s'en souvient plus, l'iniquité est brisée comme un arbre. » Rabbi Ayvo (III'$\mathrm{IV}^{\mathrm{e}} \mathrm{s}$.) fait remarquer à propos de cette dernière expression qu'il n'est pas dit «déracinée », mais «brisée ». «Comme une chose brisée, ajoute-t-il, qui produit de quoi la remplacer. Et de quoi s'agit-il ? De la génération de la tour de Babel. ${ }^{83}$ Ainsi que le dit plus loin Genèse Rabba, la génération de la tour de Babel qui a commencé deux ans après le déluge ${ }^{84} n$ 'a pas tiré les leçons de celle qui l'a précédée ${ }^{85}$.

Resh Laqish exprime différemment cette idée :

Le corbeau a donné à Noé une réponse irréfutable ${ }^{86}$, il lui a dit : « Ton maitre me hait et toi tu me hais. Ton maître me hait [car il a ordonné de prendre] sept [couples] d'animaux purs et un couple d'animaux impurs. Toi, tu me hais car tu délaisses les animaux purs et tu envoies un animal impur. Si l'ange de la chaleur ou du froid me frappait, le monde ne serait-il pas privé d'une créature ? Ou bien aurais-tu besoin de ma femelle ? $^{87}$

Par cette dernière phrase, le corbeau mis en scène par Resh Laqish fait comprendre à Noé que rien n'allait changer après le Déluge ${ }^{88}$.

Certes, aucun déluge ne s'abattra plus sur terre, mais les hommes devront rendre compte de leurs actions. Rabbi Yehoshua bar Nehemia ( $\mathrm{IV}^{\mathrm{e}} \mathrm{s}$.) interprète ainsi Gn 6, 3 : «Je ne jugerai pas leur âme seule car ils sont de chair et de sang, mais je vais leur accorder les quelques années que je leur ai fixées en ce monde puis je leur infligerai des souffrances ${ }^{89} .{ }^{90}$ Selon certains commentateurs, cette sentence fait référence au délai de 120 ans que Dieu a accordé aux

83. GnR 33, 5 (p. 310).

84. Cf. Gn 11, 10.

85. GnR 38, 2-4 (p. 352-353).

86. Jeu de mots à propos de Gn 8,7 : le corbeau envoyé par Noé «allait et revenait » yatso wa-shov. Le verbe shov signifie revenir et, dans une forme dérivée, répondre.

87. Sanhedrin $108 \mathrm{~b}$.

88. C'est ainsi que l'a expliqué Emmanuel Levinas lors d'un de ses cours hebdomadaires sur Rashi qui reprend en substance ce passage du Talmud.

89. Jeu de mots sur beshaggam « car» de Gn 6,3 et le verbe shiggem « infliger, accabler $»$.

90. GnR 26, 6 selon l'édition Romm. La version de l'édition Theodor et Albeck (p. 251) est : « Ils ne considèrent pas d'eux-mêmes qu'ils sont de chair et de sang, aussi vais-je leur accorder les années auxquelles j'ai réduit leur vie du fait de ma colère, puis je leur infligerai des souffrances. » 
hommes avant d'envoyer le Déluge. Pour d'autres, il s'agit de la vie humaine qui ne peut dépasser 120 ans et au-delà de laquelle chaque homme sera jugé corps et âme et subira le châtiment qu'il mérite.

Le mot beshaggam déjà évoqué fait dire à Rabbi Ayvo : «Qu'est-ce qui les a amenés à se révolter contre moi ? N'est-ce pas parce que je ne leur ai pas infligé de souffrances? Qu'est-ce qui maintient la porte ? Ses charnières. ${ }^{91} \mathrm{D}^{\prime}$ 'autres textes ${ }^{92}$ considèrent aussi que la génération du Déluge a péché en raison du bien-être dont elle jouissait et dont, selon les rabbins, parle Job (21, 8-15) :

Leur postérité est fortement établie devant eux, avec eux et leurs descendants sont là sous leurs yeux. Leurs maisons sont en paix, à l'abri de toute crainte; la verge de Dieu ne les atteint pas. Leurs taureaux fécondent à coup sûr ; leurs génisses mettent bas et ne perdent pas leurs petits. Ils envoient dehors leurs gamins comme un troupeau de brebis, et leurs enfants se livrent à leurs ébats. Ils entonnent des chants en s'accompagnant du tambourin et de la cithare, et ils se réjouissent au son de la flûte. Ils consument leurs jours dans le bonheur et, en un instant, ils descendent au Cheol. Et pourtant ils disent à Dieu : «Éloigne-toi de nous, nous ne voulons pas connaitre tes voies. Qu'est-ce que le Tout-Puissant pour que nous le servions ? Quel profit aurions-nous à lui adresser nos prières? »

Dorénavant, les souffrances vont donc «maintenir»l'homme comme les charnières une porte et dans cette optique, Gn. 8, 22 prend un sens tout à fait particulier. "Tant que durera la terre, semailles et moisson, froidure et chaleur, été et hiver, jour et nuit ne cesseront plus. »

« Semailles et moisson » : les hommes devront travailler la terre tous les ans alors qu'il leur suffisait auparavant de semer une fois tous les quarante ans. Ils voyaient leur descendance jusqu'à la cinquième et la sixième génération, ils engendreront ${ }^{93}$ des enfants mais les enterreront ${ }^{94}$.

« Froidure et chaleur» alors qu'avant le climat était printanier toute l'année ${ }^{95}$. Les hommes seront atteints par la fièvre et les maladies (frissons, phtisie, exanthème) ${ }^{96}$. Ils souffriront du feu et

91. Ibid. (p. 251-252). Même jeu de mots que plus haut mais également ici avec shegem « charnière ».

92. GnR 34, 11 (p. 322) ; 36, 1 (p. 334-336) ; Sanhedrin 108a ; Tan Bereshit 12 et Noah 7 ; TanB Bereshit 40 (p. 26-27).

93. Zera' signifie à la fois semence et descendance.

94. GnR 34, 11 (p. 322); Tan Bereshit 12 ; TanB Bereshit 40 (p. 26-27).

95. GnR 34, 11 (p. 322).

96. Ibid., Tan Bereshit 12. GnR 34, 11 rapporte que Rabbi Shmuel ben Nahman $\left(I V^{e}\right.$ s.) qui souffrait de maux de tête disait : "Que nous a fait la génération du déluge ?». 
de la neige alors qu'ils ne connaissaient aucun tourment ${ }^{97}$. Ils seront châtiés (au jour du jugement) par le feu et la neige ${ }^{98}$.

«Été et hiver»: les hommes seront désormais la proie des rapaces. Cette affirmation est justifiée par Is 18, $6:$ « Les rapaces passeront l'été sur eux et tous les animaux de la terre y passeront l'hiver $»^{99}$. Ils subiront les rigueurs de l'été (mitqayyetsin) et leurs visages en seront vieillis (mitharefin) ${ }^{100}$.

« Jour et nuit » : ils n'auront aucun repos ${ }^{101}$.

Nous n'avons pas épuisé ici tous les textes de la aggada concernant Noé ${ }^{102}$. Nous avons cependant pu voir à travers les exemples traités comment elle fait preuve d'une grande liberté à l'égard de la Bible. Elle amplifie le texte biblique auquel elle rajoute des récits ou d'anciennes traditions. Elle l'interprète en procédant par jeux de mots, par rapprochement de textes, par « réécriture » d'un verset. Elle s'appuie sur l'emploi d'un mot plutôt que d'un autre, sur les différents sens d'un même terme, sur la structure d'un verset...

Si l'on revient à notre sujet, l'image ambivalente que donne la aggada de Noé fait de lui un personnage plus complexe que dans la Bible et d'une certaine manière plus humain, plus proche. La aggada, en effet, s'adresse à ses contemporains auxquels elle explique le monde (les souffrances inévitables). Elle les met en garde contre les fautes les plus graves dont elle charge les hommes avant le Déluge, mais également - et peut-être surtout - les plus

97. TanB Bereshit 40 (p. 27).

98. Tan Bereshit 12.

99. GnR 34, 11 (p. 322).

100. Tan Bereshit 12. Mitharefin est à rapprocher de horef «hiver» et «vieillesse » (sens rare). Nous proposons cette lecture en fonction du contexte. Cependant, le mot mitqayyetsin n'apparaît que dans ce texte, ce qui rend la phrase difficile à comprendre. Eliezer Ben Yehuda propose, mais sans certitude : « être tourmenté par les ronces » et «avoir le visage couvert de honte " (Millon ha-safa ha- 'ivrit ha-yeshana we-ha-hadasha, t. 12, p. 5923 col. 2, n. 1). Marcus Jastrow opte pour « se sentir nauséeux » et «avoir le visage livide » (Dictionary of the Targumim, the Talmud Babli and Yerushalmi and the Midrashic Literature, p. 505).

101. Ibid. Nous avons là une autre lecture de la fin du verset, le verbe employé yishbotu signifiant à la fois cesser et se reposer.

102. Pour un recueil exhaustif des traditions juives relatives à Noé, voir Louis Ginzberg, Les Légendes des Juifs, op. cit. Voir aussi Michael E. Stone, Aryeh Amihay, and Vered Hillel (eds.), Noah and His Book(s), Atlanta, Society of Biblical Literature, 2010 ; en particulier Aryeh Amihay, « Noah in Rabbinic Literature » (p. 193-214). 
anodines (le vol de lupins) et dont seul Dieu demandera compte. Elle exprime en effet une foi absolue en la justice divine à laquelle rien ni personne n'échappe, ni l'homme, ni « le chien ou le loup », « ni le bâton ou le fouet », ni même « les arbres stériles » ${ }^{103}$.

jules.danan@inalco.fr

103. GnR 26, 6 (p. 251). 